\title{
Influence of the binder filtration on residual stresses at the composite constructions manufacturing
}

\author{
Andrei Sergeev ${ }^{* 1}$, Robert Turusov ${ }^{1,2}$, Arkadiy Gorenberg ${ }^{1}$ and Alexandr Kuperman ${ }^{1}$ \\ ${ }^{1}$ Semenov Institute of Chemical Physics, Russian Academy of Sciences, Moscow, Russia \\ ${ }^{2}$ Moscow State University of Civil Engineering, Yaroslavskoe shosse, 26, Moscow, 129337, Russia
}

\begin{abstract}
Stress changes generated during the composite winding on the mandrel surface is investigated. A model of liquid binder filtration through the fibrous filler in the form of disc-shaped gap in the structure of the coilprocessing material is proposed. An equation, considering the stress decrease occurring in consequence of the coils winding due to binder filtration is suggested. Experimental results of unidirectional rings winding on mandrels studied using strain gages are demonstrated.
\end{abstract}

\section{Introduction}

Currently polymer composite materials have found the application in construction branch by production of a timbering and fittings for buildings, profiles of various form and crosssections, sandwich panels, elements of bridges, isogrid stiffened structures and difficult architectural objects. [1-3]. At the same time winding of thread remains to one of effective ways of manufacturing designs including large-scale objects [3] today. The bearing capacity of designs, as we know, is connected with the residual tension which is saved up in a product at manufacturing. However forecasting of a coil-processing product resource is very problematic because properties and the nature of interaction of a matrix and the reinforcing material change at various stages of composite manufacturing. The aim of the work is the research of residual stresses in the wound composite at various stages of it producing.

\section{Problem statement}

Composite shell formation on a mandrel usually includes the following stages [4]: windings of binder-impregnated reinforcing element, its warming up to some elevated temperature and subsequent binder polymerization. At the step of shell winding around a cylindrical surface of liner stretching tension $\sigma^{*}{ }_{\varphi}$ is applied to the tape, fiber or fabric. Each successive coil compresses the previous one with the effort, proportional to the tension $\sigma^{*}{ }_{\varphi}$. Considering the winding of a composite on a mandrel of finite stiffness as consecutive tight fitting of extremely thin anisotropic ring on the mandrel first and then each subsequent ring

* Corresponding author: $\underline{\text { sergeevandrey89@gmail.com }}$ 
adding to the previous thickness, one can obtain the distribution of the radial and circular stresses across the thickness of the resulting rings $[4,5]$ :

$$
\begin{gathered}
\sigma_{r}(r, R(t))=\left(r^{k-1}+\frac{B}{r^{k+1}}\right) \cdot \int_{r}^{R(t)} \frac{\sigma_{\varphi}^{*} d \xi}{\xi^{k}+B \cdot \xi^{-k}}, \\
\sigma_{\varphi}(r, R(t))=k \cdot\left(r^{k-1}-\frac{B}{r^{k+1}}\right) \cdot \int_{r}^{R(t)} \frac{\sigma_{\varphi}^{*} d \xi}{\xi^{k}+B \cdot \xi^{-k}}+\sigma_{\varphi}^{*}(r), \\
\text { where } \\
B=\frac{A \cdot b^{k-1}}{A_{0}}, \\
A=b^{k-1}\left[\left(b^{2}-a^{2}\right)-b^{2} A_{0}\right], \\
A_{0}=\frac{E_{\varphi}}{2 k} \cdot\left[\frac{1-v_{0}}{E_{0}}+\frac{1+v_{0}}{E_{0}} \cdot \frac{a^{2}}{b^{2}}+\frac{k+v_{r \varphi}}{E_{\varphi}}\left(1-\frac{a^{2}}{b^{2}}\right)\right], \\
k=\sqrt{\frac{E_{\varphi}}{E_{r}}} .
\end{gathered}
$$

Here $r$ is the current radius in the composite coiled around a mandrel; $a, b$ are internal and external radii of a mandrel respectively; $E_{o}$ is the Young's modulus of a mandrel; $v_{\mathrm{o}}$ is Poisson's ratio of a mandrel; $E_{\varphi}$ и $E_{r}$ are the Young's modules of a composite in the circular and radial directions; $k$ is the coefficient of elastic anisotropy of a composite; $v_{r_{\varphi}}$ is one of Poisson's ratios of anisotropic composite; $R(t)$ is variable (increasing) external radius of the coiling composite; $t$ is time; $\sigma^{*}{ }_{\varphi}$ is defined fiber tension, generally variable, i.e. $\sigma^{*}{ }_{\varphi}(r, t)$.

It should be noted that this approach does not consider binder filtration during winding and gives the over-estimated values of the stresses in a ring in comparison with experiment. The matter is that actually there is a binder layer with thickness h between the turns of the reinforcing material, perceiving pressure from them. In its turn, the reinforcing material has the porous structure capable for binder penetration. Under the influence of pressure difference $\Delta \sigma_{r}$, created on the coil, the binder migrates from the space occupied before, resulting in the binder amounts reduction, coils compaction and stresses decrease.

To describe the migration process (filtration) in details let's imagine a model of a composite comprising a mandrel 0 impermeable for the binder, the binder layer 1 with thickness $\mathrm{s}$ and filler with the set of pores in the form of a disk-shaped gap with thickness $2 \mathrm{~h}$ [6] (fig. 1). As soon as the mandrel is impermeable to the binder, the filtration will occur in a direction perpendicular to the laying of the fibers and in the direction of the generatrix of the cylindrical surface of the coiling composite. The second case is typical only for open edges of loosely wound shell, and in case of winding on laboratory mandrels the binder filtration along the generatrix of the cylindrical surface is impossible due to the installation of intermediate disks forming a trough for winding rings. Therefore we consider that the binder moves strictly along the normal to the mandrel surface under the influence of pressure difference arising in the coil.

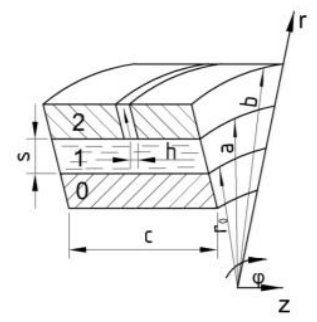

Fig. 1. The model of a binder layer (1) filtration on a mandrel (0) through a disk-shaped gap (2). 
For the three-dimensional non-stationary, laminar flow of liquid in cylindrical system of coordinates $(r, \varphi, z)$ the equations are [7]:

$$
\begin{aligned}
& \rho \cdot\left(\frac{\partial v_{r}}{\partial t}+v_{r} \cdot \frac{\partial v_{r}}{\partial r}+\frac{v_{\varphi}}{r} \cdot \frac{\partial v_{r}}{\partial \varphi}+v_{z} \cdot \frac{\partial v_{r}}{\partial z}-\frac{v_{\varphi}^{2}}{r}\right)=-\frac{\partial p}{\partial r}+\eta \cdot\left[\nabla^{2} \cdot v_{r}-\frac{v_{r}}{r^{2}}-\frac{2}{r^{2}} \cdot \frac{\partial v_{\varphi}}{\partial \varphi}\right], \\
& \rho \cdot\left(\frac{\partial v_{\varphi}}{\partial t}+v_{r} \cdot \frac{\partial v_{\varphi}}{\partial r}+\frac{v_{\varphi}}{r} \cdot \frac{\partial v_{\varphi}}{\partial \varphi}+v_{z} \cdot \frac{\partial v_{\varphi}}{\partial z}-\frac{v_{r} \cdot v_{\varphi}}{r}\right)=-\frac{\partial p}{\partial \varphi} \cdot \frac{1}{r}+\eta \cdot\left[\nabla^{2} \cdot v_{\varphi}+\frac{2}{r^{2}} \cdot \frac{\partial v_{r}}{\partial \varphi}-\frac{v_{\varphi}}{r^{2}}\right], \\
& \rho \cdot\left(\frac{\partial v_{z}}{\partial t}+v_{r} \cdot \frac{\partial v_{z}}{\partial r}+\frac{v_{\varphi}}{r} \cdot \frac{\partial v_{z}}{\partial \varphi}+v_{z} \cdot \frac{\partial v_{z}}{\partial z}\right)=-\frac{\partial p}{\partial z}+\eta \cdot \nabla^{2} \cdot v_{z}, \\
& \nabla^{2}=\frac{\partial^{2}}{\partial r^{2}}+\frac{1}{r} \cdot \frac{\partial}{\partial r}+\frac{1}{r^{2}} \cdot \frac{\partial^{2}}{\partial \varphi^{2}}+\frac{\partial^{2}}{\partial z^{2}} .
\end{aligned}
$$

where $\rho$ is density; $\eta$ is dynamic viscosity of the binder; $v_{r}, v_{\varphi}, v_{z}$ are radial, angular and axial components of the flow rate vector respectively.

The continuity equation is:

$$
\frac{1}{r} \cdot \frac{\partial}{\partial r}\left(r \cdot v_{r}\right)+\frac{1}{r} \cdot \frac{\partial v_{\varphi}}{\partial \varphi}+\frac{\partial v_{z}}{\partial z}=0
$$

Since the binder moves strictly along the normal to the mandrel surface in this model, then $v_{z}=v_{\varphi}=0$. In consequence of the axial symmetry:

$$
\begin{gathered}
\frac{\partial p}{\partial \varphi}=0, \\
\frac{\partial v_{i}}{\partial \varphi}=0(i=r, \varphi, z) .
\end{gathered}
$$

Assuming the steady flow of the liquid binder; ignoring the effect of volume forces and considering the flow isothermal (the density and viscosity are constant), from expression (3) we receive the following:

$$
\frac{\partial v_{r}}{\partial r}=-\frac{v_{r}}{r}
$$

Integrating (5), we get:

$$
\mathrm{v}_{r}=\frac{C_{1}(z)}{r}
$$

From (1) we derive the expression:

$$
-\frac{\partial p}{\partial r}+\frac{\eta}{r} \cdot\left[\frac{\partial}{\partial r}\left(r \frac{\partial v_{r}}{\partial r}\right)\right]+\eta \frac{\partial^{2} v_{r}}{\partial z^{2}}-\eta \frac{v_{r}}{r^{2}}=0 .
$$

Substituting (5) and (6) in equation (7), we obtain:

$$
-\frac{\partial p}{\partial r}+\eta \frac{\partial^{2} v_{r}}{\partial z^{2}}=0
$$

Integration of this equation twice on $d z$ and substitution of boundary conditions: $v_{r}( \pm h)=0$ gives:

$$
\mathrm{v}_{r}(r, z)=\frac{\partial p}{\partial r} \cdot \frac{z^{2}-h^{2}}{2 \eta}
$$

By substitution of (9) in (8) and integration on $d r$, we receive:

$$
p(r)=\frac{C_{1}(z) \cdot 2 \eta}{z^{2}-h^{2}} \cdot \ln r+C_{3} .
$$

Integration the equation on $d r$ and taking in account

$$
\begin{gathered}
C_{4}=\frac{C_{1}(z) \cdot 2 \eta}{z^{2}-h^{2}}, \\
p(a)=p_{0},
\end{gathered}
$$




$$
\begin{gathered}
p(b)=p_{1} \\
\text { gives: } \\
p(r)=\frac{1}{\ln \left(\frac{b}{a}\right)} \cdot\left[\left(p_{1}-p_{0}\right) \ln r+p_{0} \ln b-p_{1} \ln a\right] .
\end{gathered}
$$

Substituting (10) in (9):

$$
\mathrm{v}_{r}(r, z)=\frac{\left(p_{1}-p_{0}\right)}{2 r \cdot \eta \cdot \ln \left(\frac{b}{a}\right)} \cdot\left[z^{2}-h^{2}\right],
$$

$a \leq r \leq b$.

where $h$ is a half of the disk-shaped gap thickness; $a, b$ are respectively internal and external radii of the coiled turn of the reinforcing material, $\eta$ is dynamic viscosity of the binder; $p_{1}-p_{0}$ is the pressure difference on the chosen site, $r$ is a variable radius.

The rate of flow through the disk-shaped gap can be written in the form:

$$
Q=\int_{0}^{2 h} v_{r}(r, z) \cdot 2 \cdot \pi \cdot r \cdot d z=\frac{2 \pi \cdot \Delta p \cdot h^{3}}{3 \cdot \ln \left(\frac{b}{a}\right) \cdot \eta} .
$$

If the thickness of the reinforcing filler $\Delta=(b-a)$ is much less than the radius of winding $\mathrm{a}(\mathrm{b}-\mathrm{a}<<\mathrm{a})$, then (12) looks like:

$$
Q=\frac{2 \pi \cdot \Delta p \cdot h^{3} \cdot a}{3 \cdot \eta \cdot(b-a)}
$$

Hence, the expression for the average velocity through the cross section of the gap on the radius $b$ can be written this way:

$$
\mathrm{v}_{a v}=\frac{Q}{S_{b}}=\frac{2 \pi \cdot \Delta p \cdot h^{3} \cdot a}{3 \eta \cdot(b-a)} \cdot \frac{1}{2 \pi b \cdot 2 h}=\frac{\Delta p}{(b-a)} \cdot \frac{h^{2}}{6 \cdot \eta} \cdot \frac{a}{a+\Delta}=\frac{\Delta p}{(b-a)} \cdot \frac{h^{2}}{6 \cdot \eta} .
$$

Comparing this equation with Darcy's law for the filtration rate through a porous medium:

$$
\mathrm{v}=k_{f} \frac{\Delta p}{b-a}
$$

we can isolate the expression for filtration coefficient in our model:

$$
k_{f}=\frac{h^{2}}{6 \cdot \eta} .
$$

From the condition of equal binder consumption in cross sections on radii $a$ and $b$ $Q(a)=Q(b)$ we get:

$$
\frac{2 \pi \cdot \Delta p \cdot h^{3} \cdot a}{3 \cdot \eta \cdot(b-a)}=\frac{d s}{d t} 2 \pi \cdot a \cdot c .
$$

where ds/dt is the rate of liquid level decrease in a cavity 1 .

Introducing the concept of porosity coefficient $n=V_{p} / V=2 \mathrm{~h} / \mathrm{c} \quad\left(V_{p}\right.$ - volume of a pore, $V$ - volume of the material, $2 h$ - width of a disk pore, $c$ - width of the reinforcing material (fig. 1)) and making simplifications, we obtain expression for coils compaction due to filtration during the time $\Delta t$ :

$$
d s=\frac{\Delta p \cdot h^{2} \cdot n}{6 \cdot(b-a) \cdot \eta} d t
$$

Here is Hooke's law for an orthotropic body in case of plane stress:

$$
\left\{\begin{array}{l}
e_{r}=\frac{1}{E_{r}}\left(\sigma_{r}-v_{\varphi r} \sigma_{\varphi}\right), \\
e_{\varphi}=\frac{1}{E_{\varphi}}\left(\sigma_{\varphi}-v_{r \varphi} \sigma_{r}\right) .
\end{array}\right.
$$

Cauchy's equations in cylindrical coordinates are: 


$$
\begin{aligned}
& \varepsilon_{r}=\frac{d U}{d r}, \\
& \varepsilon_{\varphi}=\frac{U}{r} .
\end{aligned}
$$

Neglecting the radial stress contribution in the circumferential strain in the winding coils of a composite, from (15) and (16) we receive:

$$
d U(b)=\frac{b \cdot d \sigma_{\varphi}}{E_{\varphi}} .
$$

The liquid level decrease (ds) in radial direction in (14) is equal to coil compaction in radial direction $d U$ upon the influence of tension $\sigma_{\varphi}$ in expression (17):

$$
d s=d U
$$

From the equilibrium conditions of the composite coil we conclude that pressure difference in the binder is the same as in the coil:

$$
\Delta p=\Delta \sigma_{r}=-\frac{\sigma_{\varphi}^{*} \cdot(b-a)}{b} .
$$

After the substitution of (17) and (19) in (14) and keeping in mind (13) and (18) we get:

$$
\frac{d \sigma_{\varphi}(b, t)}{d t}=-\frac{k_{f} \cdot n \cdot E_{\varphi}}{b^{2}} \cdot \sigma_{\varphi}^{*}(b, t) .
$$

Solving the equation (20), we obtain the expression for stresses decrease during winding, similar to those in [8]. However, the approach presented here allows evaluating the filtration coefficient structurally:

$$
\begin{gathered}
\sigma_{\varphi}(b, t)=\sigma_{\varphi}^{*}(t) \cdot e^{-A \cdot t}, \\
\text { where } \\
A=\frac{E_{\varphi} \cdot K_{f}}{b^{2}}, \\
K_{f}=k_{f} \cdot n=\frac{h^{2} \cdot n}{6 \cdot \eta} .
\end{gathered}
$$

Here $h$ is half of the height of the disc gap; $b$ is external radius of the wound coil of the reinforcing material (variable during winding), $\eta$ is dynamic viscosity of the binder; $n$ is porosity coefficient of the reinforcing material.

At the stage of heating the binder pressure difference in the known temperature field changes by

$$
\Delta p^{\prime}=\alpha \cdot \Delta T \cdot E
$$

Here $\alpha$ is a coefficient of binder linear expansion; $\Delta \mathrm{T}$ is a difference between the current and winding temperatures; $E$ is modulus of binder elasticity (Young's modulus). Binder warming results in its viscosity decrease, leading to the increase of liquid binder consumption from the previously occupied space and superior composite coil compaction:

$$
d U=\frac{(\Delta p+\alpha \cdot \Delta T \cdot E) \cdot h^{2} \cdot n}{6 \cdot(b-a) \cdot \eta} d t
$$

Thus, the rate of binder filtration through the filler increases with the rising temperature, resulting in residual stresses drop. Binder viscosity reduction due to heating also leads to the decrease of the stresses generated at the step of winding.

\section{Test procedure and experiment}

Laboratory tests with strain gages were carried out to study the stress-strain state of composite shell in different solidification techniques. 
Test samples were segments of the steel pipe with a diameter of $56 \mathrm{~mm}$, thickness of 3 $\mathrm{mm}$ and length of $75 \mathrm{~mm}$. Strain gages with the base length of $10 \mathrm{~mm}$ were installed on the outer and inner surface of the pipe in the circumferential direction. Multichannel strain test station SIIT-3 was used for signal registration. Calibration of the instrument showed that one division of the scale corresponds to deformation $\varepsilon=10^{-6}$.

Before winding the mandrel was installed in the centers of the coil-processing machine, so that it could be freely deformed in the axial and circumferential directions by the action of winding thread tension. The thread was aramide Rusar-S fiber (two threads 58,8 tex each). Tension of the winding thread was specified by the device creating braking of the impregnating roller in the coil-processing machine. Several experiments were carried out; the fiber tension therein was constant over time, the winding rate was $45 \mathrm{rpm}$ with the step $0,75 \mathrm{~mm} /$ revolution.

Two experiments were conducted. The first one was with epoxy binder EDT-10 and hot solidification technology curing agent - triethanolamine titanate (TEAT) in the quantity of 10 mass percent. In this case the thread tension was $60 \mathrm{~N}$, (i.e. the tensile stress of each thread was $75 \mathrm{kgs} / \mathrm{mm}^{2}$, which is about $14 \%$ of the fiber rupture durability). In the second case the same oligomer was tested with cold solidification technology binder polyethylenepolyamine (PEPA) in the quantity of 7,5 mass percent. Here the winding thread tension was $80 \mathrm{~N}$ (tensile stress of each thread was $100 \mathrm{kgs} / \mathrm{mm}^{2}$, i.e. $19 \%$ of the fiber rupture durability). Prior to the end of the winding at the shell thickness of about 2 $\mathrm{mm}$ one more strain gages and Chromel-Copel thermocouple were positioned in the circumference of the organic plastic structure to monitor stress changes of the material during sample solidification.

In the first experiment strain gages data were registered during thread winding on the mandrel. Fig. 2 shows the theoretical curve 1 which was built using equation (1); and experimental values obtained from strain gages installed on outer and inner radii of the pipe (marked with white and shaded circles on fig. 2). Resulting values of the strains can be recalculated into compressive stress $\sigma_{b o r}$, rendered by winding on a steel mandrel:

$$
\sigma_{b o r}=2 \frac{e_{\varphi}(r) \delta E}{D_{a v}} .
$$

Here $e_{\varphi}$ is the measured strain; $\delta$ is ring thickness; $D_{a v}$ is average diameter of the pipe; $E$ is Young's elasticity modulus of the steel pipe.

Let's assume that the discrepancy between the theoretical curve and the experimental values of the stress is associated only with binder filtration through a fiber. In this case we substitute expression (21) in (1). Now we can find the estimated filtration coefficient, so that the tension on a mandrel dependence on number of coils satisfies the experiment (curve 2, fig. 2.). This filtration coefficient is $K_{f}=0,065 \mathrm{~mm}^{4} / \mathrm{kgs} \cdot \mathrm{s}$.

In the second experiment sensor data registration in the intermediate stages of winding was not carried out, at the end of winding internal sensor showed deformation $\varepsilon=0,0037$.

Further studies have shown that the tension on the mandrel remains constant at room temperature for a long time and it does not depend on whether the binder is polymerized or not.

Next, measurements of the composite tension on the mandrel during the heat treatment process were carried out. Temperature conditions of heat treatment in both cases were as follows: heating up to the temperature of $160{ }^{\circ} \mathrm{C}$ for 2 hours followed by the exposure at this temperature for 5 hours. Quartz plate glued with strain gages and a thermocouple was incubated in the same conditions as the test sample in order to take into account the temperature-derived errors of strain gages indications. A deformation of the quartz plate can be ignored, so the temperature dependence of the data coming from quartz plate bound 
sensor can be considered as the correction to the indications of the sensors attached to the sample. It was about 5 divisions per $1^{0} \mathrm{C}$.

Figure 3 demonstrates the data changes of strain gages positioned on the border of the mandrel and a composite ring during heat treatment of the sample based on hot solidification binder. Site 1 shows the mandrel compression during the winding, site 2 heating of the sample, viscosity decrease of the binder and then viscosity increase during polymerization. All these steps lead to almost complete disappearance of residual compressive stress. Some stretching stresses occur on the border of the ring and the mandrel and persist up to a certain temperature in the area 3. These stretching stresses can be explained by the fact that the mandrel is associated by adhesive connection with the shell, whose thermal expansion coefficient is lower than the one of steel. The following polymerized (solid) samples heating and cooling cycles are reversible (Figure 4), and they have little effect on the value of residual strain.

In the second assay samples based on cold solidification technology binder were subjected to the same heat treatment as in the first experiment. However, in this case, the composite shell was precured at $50^{\circ} \mathrm{C}$ for 24 hours. The dependencies in this case were generally as in the previous experiment, but the decrease of the compressive stress on the mandrel was only $3 \%$ (fig. 5). Heat treatment of the sample is due to additional binder solidification during heating and its softening at the temperatures above the glass transition temperature of this polymer.

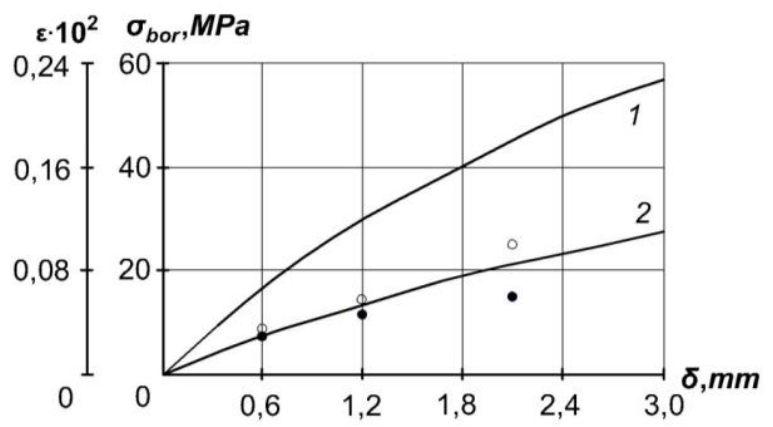

Fig.2. Deformation $\varepsilon$ and stresses $\sigma_{r}=P$ on the border of the winding ring and mandrel depending on winding thickness $\delta$. 1-curve built ignoring the filtration; 2-experimental curve $\left(\mathrm{K}_{\mathrm{f}}=0,065 \mathrm{~mm} / \mathrm{kgs} \cdot \mathrm{s}\right.$, details are given in the text)

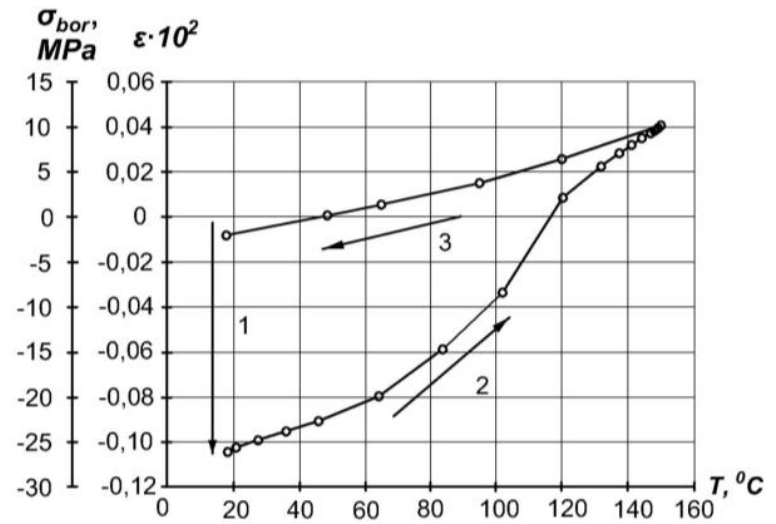

Fig. 3. Deformation of the strain gages installed on the inner surface of the pipe (hot solidification technology) 


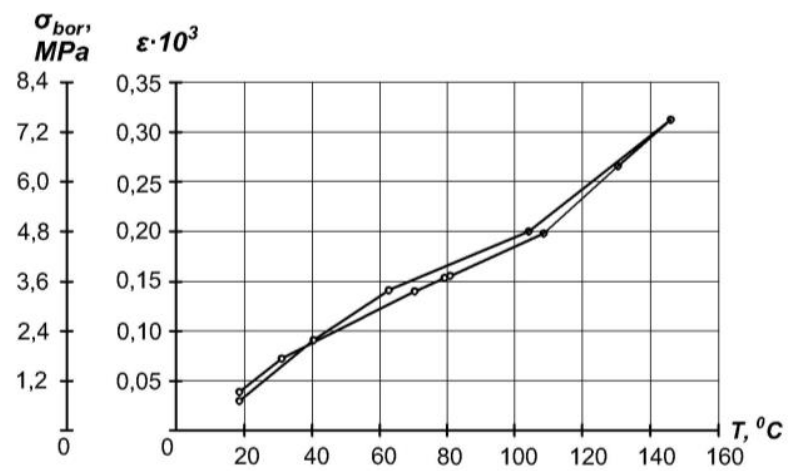

Fig. 4. Deformation of the strain gages installed on the inner surface of the pipe during repeated cooling-heating of the sample obtained using hot solidification technology binder.

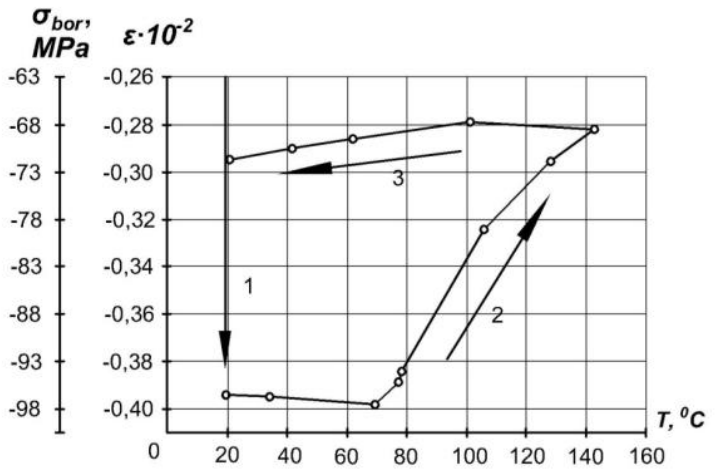

Fig. 5. Deformation of the strain gages installed on the outer surface of the pipe (cold solidification technology)

\section{Conclusion}

So, it is established that winding, heating and hardening stage is followed by binder filtration through porous filler structure, to the top layers of a coil-processing product at thick-walled composite forming. Filtration process considerably limits possibilities of creation of favorable residual stresses at a stage of winding and leads to considerable falling of the compressed stresses as a result of a warming up and hardening. At the same time as a result of filtration in some layers of a composite there can be a binder deficiency. In the case of established adhesive interaction between binder and fiber binder becomes comprehensively stretched at the microlevel, it leads to decrease in tightness of a composite product. For receiving qualitative coil-processing constructions it is necessary to consider technology of hardening binder.

\section{References}

1. Zhu D., Shi H., Fang H., Liu W., etc. Fiber reinforced composites sandwich panels with web reinforced wood core for building floor applications // Composites Part B, , doi: 10.1016/j.compositesb.2018.05.048, (2018)

2. Sonnenschein R., Gajdosova K., Holly I. FRP composites and their using in the construction of bridges // Procedia Engineering 161, pp. 477 - 482, ( 2016 ) 
3. Riccardo La Magna, Frederic Waimer, Jan Knippers Coreless winding and assembled core - novel fabrication approaches for FRP based components in building construction. Construction and building materials, http://dx.doi.org/10.1016/j.conbuildmat.2016.01.015, (2016)

4. Sergeyev A. Yu., Turusov R.A., Baurova N.I., Kuperman A.M. Stresses arising during cure of the composite wound on the cylindrical surface of an element of exhaust system. Mechanics of Composite Materials, V. 51, No. 3, pp. 321-332, (2015)

5. Elakov A.B., Turusov R.A., Bogachev E.A., Sergeev A.Yu. Simulation of the stressstrain state in non-woven fabric winding onto a cylindrical mandrel, St. Petersburg polytechnic university of engineering science and technology, 24(02), pp. 149-160, DOI:10.18721/JEST.240213, (2018)

6. Turusov R.A., Rouabhi A. Capillary model of a wound thick-walled cylinder. Mechanics of Composite Materials V. 50, I. 1, 65-70 (2014)

7. Loytsyansky L.G. Mechanics of liquid and gas (Dropha, Moscow), (2003)

8. V. V. Bolotin, A. N. Vorontsov, R. Kh. Murzakhanov, "Analysis of the technological stresses in wound components made out of composites during the whole duration of the preparation process," Mech. Compos. Mater., 16, No. 3, pp. 361-368 (1980) 